\title{
Emergency Medical Services Capacity for Prehospital Stroke Care in North Carolina
}

Mehul D. Patel, MSPH; Jane H. Brice, MD, MPH; Kelly R. Evenson, PhD; Kathryn M. Rose, PhD; Chirayath M. Suchindran, $\mathrm{PhD}$; Wayne D. Rosamond, PhD

Suggested citation for this article: Patel MD, Brice JH, Evenson KR, Rose KM, Suchindran CM, et al. Emergency Medical Services Capacity for Prehospital Stroke Care in North Carolina. Prev Chronic Dis 2013;10:130035. DOI: http://dx.doi.org/10.5888/pcd10.130035 圈.

PEER REVIEWED
In this audio podcast, listen to lead author and PCD's 2013 Student Research Paper Contest winner, Mehul D. Patel, talk about stroke care and emergency medical services.

\section{Abstract}

\section{Introduction}

Prior assessments of emergency medical services (EMS) stroke capacity found deficiencies in education and training, use of protocols and screening tools, and planning for the transport of patients. A 2001 survey of North Carolina EMS providers found many EMS systems lacked basic stroke services. Recent statewide efforts have sought to standardize and improve prehospital stroke care. The objective of this study was to assess EMS stroke care capacity in North Carolina and evaluate statewide changes since 2001.

\section{Methods}

In June 2012, we conducted a web-based survey on stroke education and training and stroke care practices and policies among all EMS systems in North Carolina. We used the McNemar test to assess changes from 2001 to 2012.

\section{Results}

Of 100 EMS systems in North Carolina, 98 responded to our survey. Most systems reported providing stroke education and training (95\%) to EMS personnel, using a validated stroke scale or screening tool (96\%), and having a hospital prenotification policy (98\%). Many were suboptimal in covering basic stroke educational topics (71\%), always communicating stroke screen results to the destination hospital (46\%), and always using a written destination plan (49\%). Among 70 EMS systems for which we had data for 2001 and 2012, we observed significant improvements in education on stroke scales or screening tools $(61 \%$ to $93 \%, P<.001)$ and use of validated stroke scales or screening tools $(23 \%$ to $96 \%, P<.001)$.

\section{Conclusion}

Major improvements in EMS stroke care, especially in prehospital stroke screening, have occurred in North Carolina in the past decade, whereas other practices and policies, including use of destination plans, remain in need of improvement.

\section{Introduction}

Emergency medical services (EMS) can have a positive impact on the care of acute stroke patients through early identification and expedited transport and thus more timely delivery of treatments, notably thrombolytic therapy (1). With proper education and use of protocols, EMS personnel can screen for stroke in the field, initiate patient evaluation, and transport patients to a specialized stroke center (2-4). However, current levels of EMS education and prehospital care practices for stroke patients are not well characterized and vary by location (5-7).

Improving EMS capabilities to respond to and manage care of acute stroke patients is important because stroke is a major cause of death and disability in the United States (8). In North Carolina, state legislation was passed in 2006 to address the availability of stroke-related resources among hospitals and EMS systems (9). This legislation led to the 
development and implementation of standardized EMS stroke care practices and policies. By 2010, all EMS systems in North Carolina were required to use a standardized protocol to guide the prehospital care of stroke patients and a written destination plan to facilitate the transport of stroke patients to the most appropriate hospital.

A statewide survey in 2001 of EMS stroke care in North Carolina found EMS education and the use of protocols to be lacking (5). However, in the past 10 years, major national and statewide changes have occurred in the prehospital management and care of stroke patients, including the use of standardized protocols and validated stroke screening tools and the development and use of destination plans (10-12).

The objective of this study was to examine current EMS stroke education and stroke care practices and policies in North Carolina and to evaluate statewide changes since 2001. Given advancements in prehospital stroke care and recent EMS implementation of stroke policies, we hypothesized improvements in EMS stroke care capacity during the past decade.

\section{Methods}

\section{Study design and data collection}

We developed a 31-item web-based survey to collect information on the stroke care capacity of EMS systems in North Carolina. The survey measured frequency and educational content of stroke training sessions and information about stroke care practices and policies. Questions were adopted from other published surveys of EMS stroke care capacity $(5,6)$ or developed on the basis of expert input from 2 local EMS medical directors. We also assessed general EMS system characteristics, including pay structure and level of service. The survey instrument and methodology were approved by the institutional review board of the University of North Carolina at Chapel Hill. A copy of the survey is available at www.unc.edu/ kevenson/_2012_NC_EMS_StrokeSurvey.pdf.

The 100 county-based EMS systems in North Carolina comprise approximately 35,00o EMS personnel and 540 EMS agencies (13). We identified 100 EMS administrative directors through the state regulatory office directory and invited them to complete the web-based survey. We selected these directors as key informants because they supervise EMS personnel and manage the daily operations of their systems. Survey instructions encouraged respondents to elicit information from others in their organizations, such as training officers and medical directors. Links to the online survey were e-mailed in June 2012. We sent reminder e-mails 1 and 2 weeks after the initial invitation. We also made follow-up telephone calls to nonrespondents and offered the option to complete the survey by telephone.

\section{Data processing and analysis}

We devised a summary score of EMS stroke care capacity using parameters recommended by national and local experts $(10,12)$. Ideally, a stroke-capable EMS system should address 4 priority areas: education and training, protocol and screening, destination plan, and continuous quality improvement. Each EMS system responding to our survey was assessed for each priority area and assigned points according to our assessment. Each area was equally weighted with a maximum of 3 points, thus allowing an overall maximum stroke care capacity score of 12 points (Box).

We calculated descriptive statistics for the scores among all responding systems. Overall scores were categorized into 4 groups: o to 3 points, 4 to 6 points, 7 to 9 points, and 10 to 12 points. We compared frequencies of scores by estimated annual patient volume of the EMS system and by county population density. We estimated annual patient volume as the number of EMS events in the past year, as recorded in the North Carolina Credentialing Information System (16), and then categorized patient volume into 3 groups: fewer than 5,000 events, 5,000 to 20,000 events, and more than 20,000 events. County population density was categorized as metropolitan, micropolitan, or rural as defined by the US Office of Management and Budget (17).

In 2001, a survey mailed to 83 EMS agencies in North Carolina was completed and returned by 72 of them (5). To enable comparison between the 2012 and 2001 surveys, we repeated questions on stroke education, transport with lights and sirens on, use of validated stroke screening tools, and policy on advance notification of hospitals. We acquired the 2001 survey

\section{Box. Emergency Medical Services (EMS) Stroke Care Capacity Scoring System, North Carolina, 2012}

\begin{tabular}{|l|c|}
\hline Priority Areas and Measures & Points \\
\hline \multicolumn{2}{|l|}{ Education and training } \\
\hline $\begin{array}{l}\text { At least 2 hours of stroke training provided } \\
\text { per year }\end{array}$ & 1 \\
\hline $\begin{array}{l}\text { Personnel trained on stroke at least once } \\
\text { per year }\end{array}$ & 1 \\
\hline $\begin{array}{l}\text { Training covers basic stroke educational } \\
\text { topicsa }\end{array}$ & 1 \\
\hline Protocol and screening & 1 \\
\hline Standardized stroke protocol & 1 \\
\hline Validated stroke scale or screening toolb & 1
\end{tabular}


responses from the study authors and matched them by EMS system to our survey responses. The comparison analysis was restricted to the 70 EMS systems for which we had data for both years. We compared this subset of 70 EMS systems with all EMS systems in North Carolina by patient volume, number of EMS personnel, and level of service and found minimal differences.

We calculated both absolute and relative changes in EMS stroke care capacity measures between 2001 and 2012. A relative change greater than $10 \%$ was considered meaningful. We tested the difference between paired proportions by using the 2-sided McNemar exact test. The 2-sided Fisher exact test was used for categorical data and the Wilcoxon rank sum test for non-normal continuous data. A $P$ value less than .05 was considered significant.

\section{Results}

\section{North Carolina EMS systems}

Of 100 EMS systems in North Carolina, 2 systems provided basic life-support service only, and 98 systems provided all or some advanced life support service. The EMS systems varied by number of certified EMS personnel (median, 120 personnel; interquartile range [IQR], 66-235 personnel) and by estimated annual patient volume (median, 8,004 patients; IQR, 3,754-17,848 patients) (16). Based on county population estimates, 40 systems serviced metropolitan areas, 30 micropolitan, and 30 rural.

\section{EMS stroke survey}

We received survey responses from 98 of 100 EMS systems. Most respondents completed the survey online; 9 surveys were conducted by telephone. Primary survey respondents were administrative directors $(n=80)$, training officers (n =12), and 1 medical director; 5 respondents did not report their job title. Seven surveys had 2 respondents (eg, a director and a training officer, an administrative director and an emergency department nurse).

Most systems (95\%) provided at least 1 stroke training session to EMS personnel in the past 2 years (Table 1); of these, $74 \%$ provided stroke education at least once per year. The educational content of training sessions always included stroke signs and symptoms and frequently included stroke scales or screening tools (95\%); thrombolytic therapy was addressed less frequently (66\%). In-person classroom training sessions were almost always offered, but online courses and videos were also used. Almost all surveyed EMS systems used a validated stroke scale or screening tool, such as the Los Angeles Prehospital Stroke Screen (66\%) (3) or the Cincinnati Prehospital Stroke Scale (52\%) (2). However, only $46 \%$ reported always communicating stroke scale or screen results to the destination hospital. Similarly, only $49 \%$ reported always using a written plan to determine the destination hospital. Lastly, 98\% of EMS systems reported having a policy to notify the hospital in advance when transporting a suspected stroke patient.

\section{EMS stroke care capacity score}

Among the 98 EMS systems analyzed, stroke care capacity scores ranged from 4 to 12 points. The median score was 7 points (IQR, 6-9 points), and 3 systems scored the maximum 12 points. Most systems provided at least 2 hours of stroke training per year (78\%), educated personnel at least once per year (69\%), and covered the basic stroke educational topics (66\%); only $44 \%$ did all 3 of these activities, and $12 \%$ did none. Only $44 \%$ of systems scored the maximum 3 points for protocol and screening, and only $45 \%$ scored the maximum 3 points for destination plan, even though the state regulatory office requires each system to implement a standardized stroke protocol and written destination plan. Performance feedback was uncommon: only $13 \%$ of systems had evaluated their stroke patient care data in the past year. Overall, EMS stroke care capacity scores in North Carolina showed room for improvement (Figure 1). Although no EMS system scored fewer than 4 points overall, 30 systems scored 6 points or fewer. We observed high scores (ie, 10-12 points) in all categories of patient volume and county population density. 


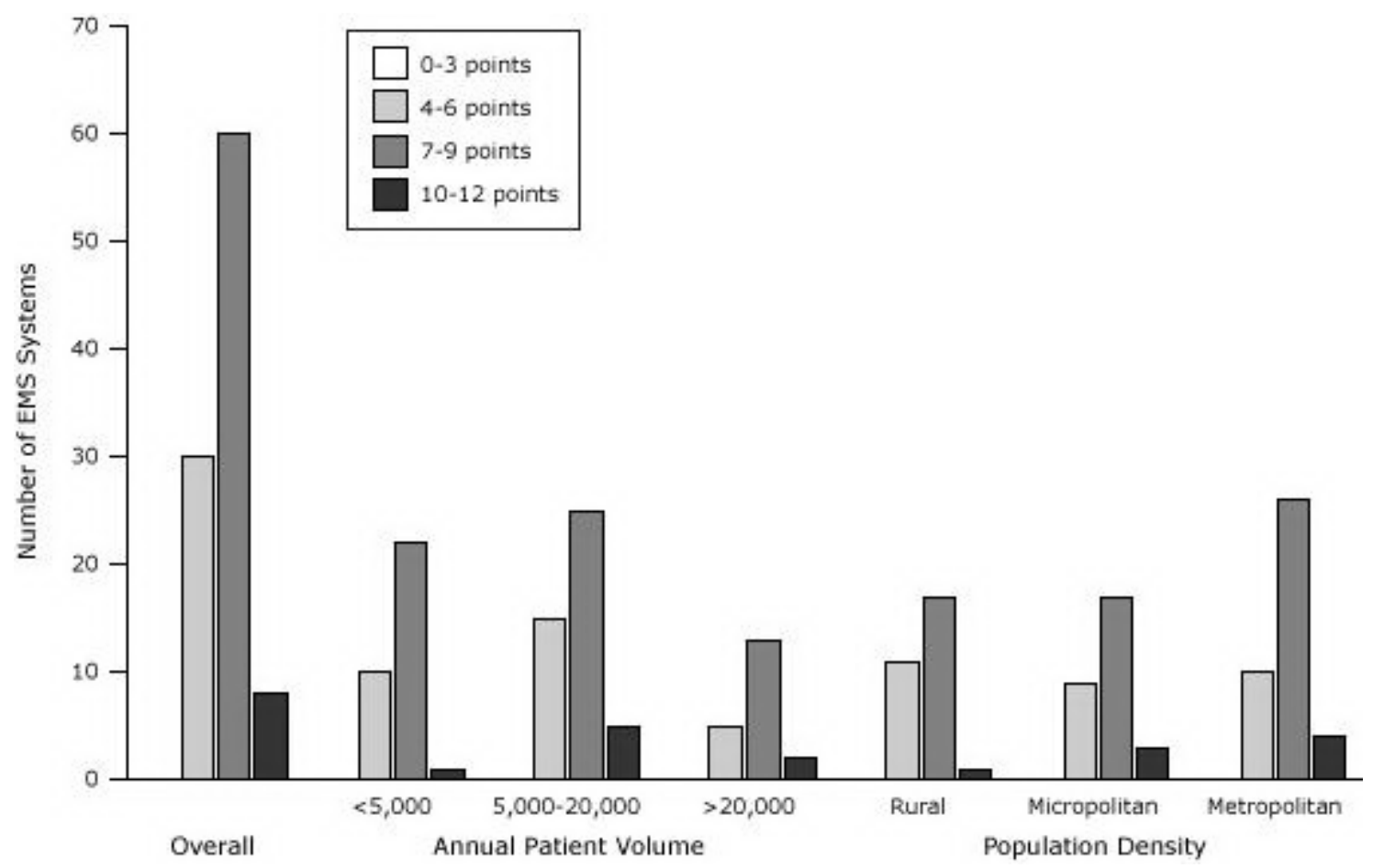

Figure 1. Emergency Medical Services (EMS) stroke care capacity scores for 98 EMS systems responding to survey, overall and by patient volume and county population density, North Carolina, 2012. No system scored 0 to 3 points. County population density was categorized as metropolitan, micropolitan, and rural as defined by the US Office of Management and Budget (17). [A tabular version of this figure is also available.]

\section{Comparison between 2001 survey and 2012 survey}

We observed a moderate increase in the percentage of EMS systems providing stroke education and in the overall median number of hours of stroke education provided (Table 2). Although the percentage of systems providing education on stroke risk factors and pathophysiology decreased, the percentage providing education on stroke signs and symptoms and thrombolytic therapy increased. We observed a significant increase ( 31 percentage points, absolute; $51 \%$, relative; McNemar exact test $=18.6, P<.001$ ) in the percentage of systems providing education on stroke scales and screening tools. Coverage of the basic 4 stroke educational topics also increased from $54 \%$ to $67 \%$. The greatest change was the increase in use of validated stroke scales or screening tools (from 23\% to 96\%; McNemar exact test = $50.0, P<.001)$. A policy to notify hospitals in advance of stroke patient arrival existed at $71 \%$ of systems in 2001 ; all systems had adopted this policy by 2012. Although18 systems changed from not covering all 4 basic educational topics to covering them and 9 changed from covering to not covering them (Figure 2), the result was a 13 percentage-point increase in the proportion of systems covering these topics. The 72 percentage-point increase in the use of validated stroke scales or screening tools was solely driven by 50 systems that changed positively. 


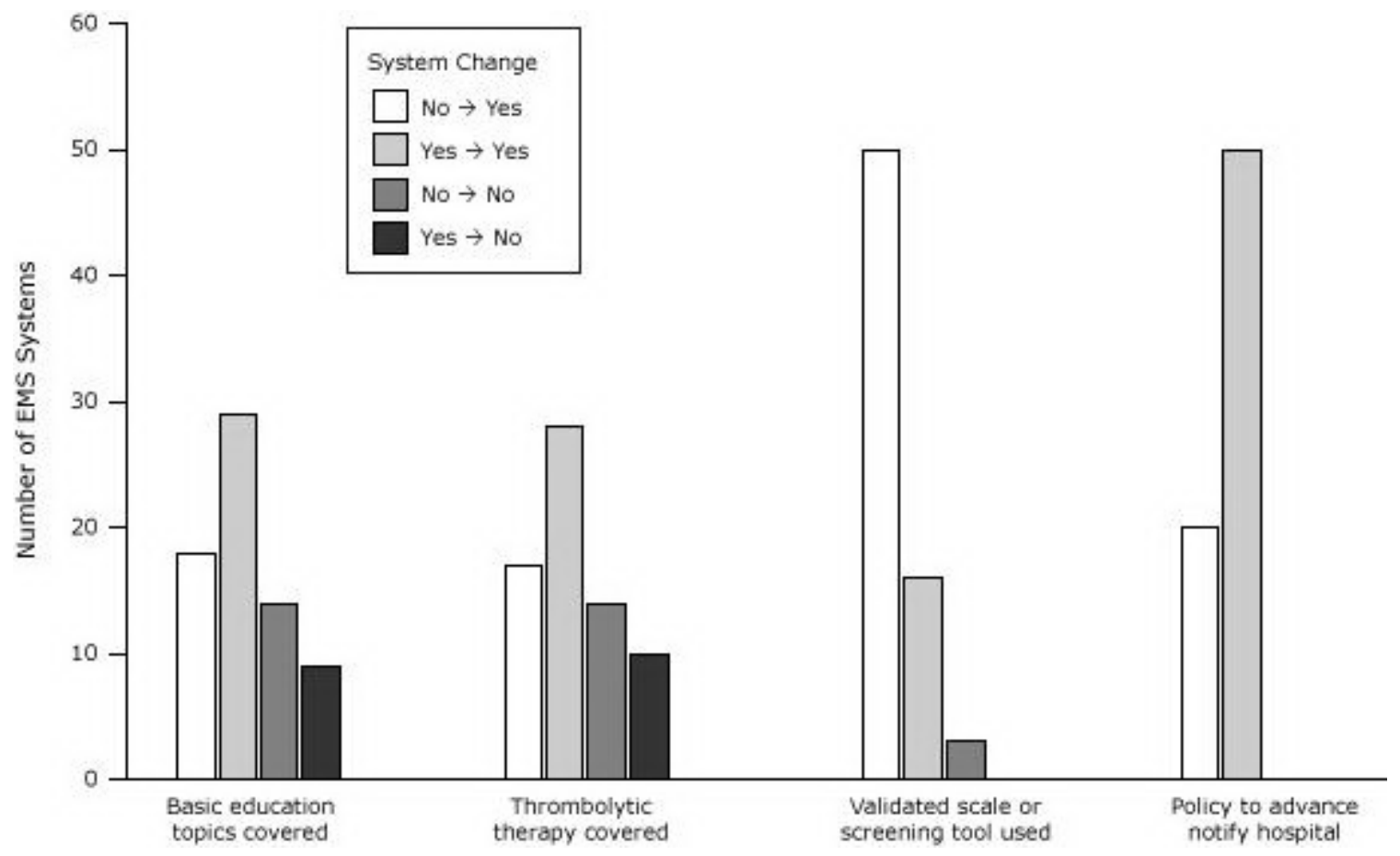

Figure 2. Changes in selected stroke care capacity measures from 2001 to 2012 among 70 Emergency Medical Services systems, North Carolina. The 70 systems participated in surveys administered in 2001 and 2012. Basic education topics were stroke risk factors, signs and symptoms, pathophysiology, and scale or screening tool. Not included in basic topics was thrombolytic therapy. One system did not answer the question on thrombolytic therapy, and one did not answer the question on use of validated scale or screening tool. [A tabular version of this figure is also available.]

\section{Discussion}

Our study found that some aspects of EMS stroke care in North Carolina were practiced almost universally, including stroke education, use of validated stroke scales or screening tools, and a policy to notify hospitals in advance of suspected stroke. However, data on other measures of stroke education and prehospital practice and policies suggested room for improvement. Among EMS systems that provided stroke training sessions, almost one-third did not cover the basic stroke educational topics. Of the systems surveyed, 69\% educated their personnel on stroke at least annually. This percentage was only moderately greater than the 60\% found in a study of EMS agencies in Minnesota in 2006, one of the few published, statewide assessments of EMS stroke care capacity (6).

Although almost all EMS systems in North Carolina used a validated stroke scale or screening tool, fewer than half regularly communicated the results to the destination hospital. This finding is consistent with the finding that only $34 \%$ of EMS agencies in Minnesota verbally communicated stroke scale findings (6). Moreover, almost all (98\%) systems in North Carolina reported a policy to notify hospitals in advance of suspected stroke patients. There appears to be an inconsistency between policy and compliance. Previous studies observed that prenotification by EMS personnel of a suspected stroke can significantly reduce in-hospital delays and increase treatment rates (18-20). Follow-up to our quantitative work could use qualitative methods or intervention studies to better understand the translation of advance notification policies into EMS communication practices.

Bypassing local community hospitals for specialized stroke centers by EMS is a recommended policy and practice for many stroke systems of care (10). Furthermore, all EMS systems in North Carolina are required to implement a destination plan for stroke. In comparison, only 37\% of EMS agencies in Minnesota reported having such a plan (6). Although a plan is required in North Carolina, our survey showed that only about half of EMS systems always used their plan and another $12 \%$ never or only sometimes used it, suggesting that even with a statewide policy, local systems are complying at varying degrees. Differences in publicizing legislation and enforcing EMS policies across the state may have had an impact on local compliance, although we did not investigate these differences.

We found room for overall improvement in EMS stroke care capacity: 92\% of systems scored less than 10 points of a possible 12. Of the main priority areas, continuous quality improvement was the least frequently addressed; only 13 systems (13\%) had examined stroke care performance data in the previous year. A Utah-based study examined the feasibility of using electronic EMS records for monitoring prehospital stroke care and found that only $58 \%$ of EMS 
agencies entered data into an electronic system and that data were often incomplete (21). EMS systems in North Carolina are required to enter standardized data elements electronically, so all systems should have the necessary data for performance feedback (12). Moreover, a statistical analysis report on stroke patient data was recently designed and developed to improve EMS systems (12). Nonetheless, we found few systems generate these reports, and more work is needed to encourage data-driven continuous quality improvement in North Carolina and in other states.

Although low patient volume and rural locations are reported to limit EMS stroke care capacity $(7,22-25)$, our comparisons by patient volume and county population density did not demonstrate strong variation by these characteristics. In fact, our findings show that some systems with low patient volume in rural areas have high stroke care capacity. However, further investigation using a larger sample size is needed to examine the relationship between EMS stroke care capacity and patient volume and county population density.

EMS capacity in North Carolina improved since 2001, especially in education and use of a validated stroke scale or screening tools, such as the Los Angeles Prehospital Stroke Screen or the Cincinnati Prehospital Stroke Screen. Our findings are consistent with those of similar studies. In 2006, 47\% of Minnesota EMS agencies reported using the Cincinnati Prehospital Stroke Screen (6), whereas a more recent study (7) found that 80\% of EMS agencies in 9 states reported using a stroke scale (although the scale used was not identified). Other significant positive changes were in education on all basic stroke topics, education on thrombolytic therapy, and a policy to notify hospitals in advance. Although we observed positive changes overall, we also found that certain EMS systems underwent negative changes (eg, in basic stroke educational topics). Although a formal policy evaluation was beyond the scope of this study, our findings show that statewide standardization of EMS stroke care was associated with improvements in capacity. Other states and regions that implement similar policies may also undergo significant improvements.

Our study has several limitations. Although our survey questions were not validated, they are similar to those in other surveys and were developed with input from subject matter experts $(5,6)$. Our results were based on self-reports and are subject to inaccurate responses. However, respondents were selected because of their knowledge of their systems; in addition, respondents could work with others in completing the survey. Only 2 of the 100 systems did not respond, and systems that did not participate were well represented by the survey respondents according to service level (ie, basic life support, advanced life support), patient volume, and county population density. Our EMS stroke care capacity score was based on expert opinion and guideline recommendations but has not been independently validated. However, it can be easily replicated in other regions, and we believe it provides a useful summary of overall EMS capacity for stroke. In the absence of guidance in published literature, we chose to weight each priority area equally. We encourage further research to improve on this scoring method. A strength of our study was the direct systemspecific comparisons between 2 years of data. Although we compared changes in 70 EMS systems only, these systems serviced about $81 \%$ of the 9.5 million residents in North Carolina. This subset of systems was similar to all EMS systems in North Carolina in level of service, patient volume, and county population density.

Our findings demonstrate areas of progress and areas that need improvement if EMS systems are to provide the best care for stroke patients in North Carolina. Education of EMS personnel should continue to be a focus, especially the content of stroke training sessions. Significant progress occurred in the institution of standardized patient care protocols, validated scales and screening tools, destination plans, and advance notification policies. However, the use of destination plans and communication to hospitals of stroke screen results need improvement. Given its large stroke burden and recent statewide actions to advance stroke care, North Carolina was a unique setting for this study. Many of the improvements observed in this study could be explained by statewide efforts to standardize prehospital stroke care and encourage best practices, such as bypassing local hospitals for stroke centers, although secular trends also likely played a role. Although other states may not require standardized protocols and destination plans, this study offers an example of how stroke care capacity can improve after such a policy is put in place. For local health services planning and quality improvement, it is important to continuously monitor the capacity of EMS systems to respond to and manage stroke patients. Further study is needed to understand how stroke capacity translates into actual EMS care received.

\section{Acknowledgments}

This research received no funding support. The authors thank the EMS personnel who completed the survey. We also thank Chailee Moss for her assistance in conducting the survey. The North Carolina Office of EMS and the North Carolina EMS Data System support state, regional and local EMS and health care-related service delivery from a patient care, resource allocation, and regulatory perspective. In preparing this manuscript, we did not collaborate with investigators of the North Carolina EMS Data System, and the manuscript does not necessarily reflect the opinions or views of the North Carolina Office of EMS, EMS Performance Improvement Center, or the study sites participating in the North Carolina EMS Data System.

\section{Author Information}


Corresponding Author: Mehul D. Patel, MSPH, Department of Epidemiology, Cardiovascular Disease Program, 137 E Franklin St, Ste 306, Chapel Hill, NC 27514. Telephone: 919-966-1967. E-mail: mehul.patel@unc.edu.

Author Affiliations: Jane H. Brice, Kelly R. Evenson, Chirayath M. Suchindran, Wayne D. Rosamond, University of North Carolina at Chapel Hill, Chapel Hill, North Carolina; Kathryn M. Rose, SRA International, Durham, North Carolina.

\section{References}

1. Schwamm LH, Pancioli A, Acker JE 3d, Goldstein LB, Zorowitz RD, Shephard TJ, et al. Recommendations for the establishment of stroke systems of care: recommendations from the American Stroke Association's Task Force on the Development of Stroke Systems. Stroke 2005;36(3):690-703. CrossRef 圈 PubMed 国

2. Kothari RU, Pancioli A, Liu T, Brott T, Broderick J. Cincinnati Prehospital Stroke Scale: reproducibility and validity. Ann Emerg Med 1999;33(4):373-8. CrossRef 圈 PubMed 圈

3. Kidwell CS, Starkman S, Eckstein M, Weems K, Saver JL. Identifying stroke in the field. Prospective validation of

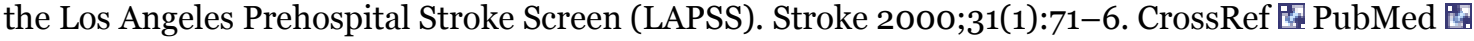

4. Gladstone DJ, Rodan LH, Sahlas DJ, Lee L, Murray BJ, Ween JE, et al. A citywide prehospital protocol increases access to stroke thrombolysis in Toronto. Stroke 2009;40(12):3841-4. CrossRef 圈 PubMed 圈

5. Brice JH, Evenson KR, Lellis JC, Rosamond WD, Aytur SA, Christian JB, et al. Emergency medical services education, community outreach, and protocols for stroke and chest pain in North Carolina. Prehosp Emerg Care 2008;12(3):366-71. CrossRef 圈 PubMed 圈

6. Tsai AW. Prehospital and emergency department capacity for acute stroke care in Minnesota. Prev Chronic Dis 2008;5(2):A55. PubMed 圈

7. Greer S, Williams I, Valderrama AL, Bolton P, Patterson DG, Zhang Z. EMS medical direction and prehospital practices for acute cardiovascular events. Prehosp Emerg Care 2013;17(1):38-45. CrossRef $\mathrm{O}_{\mathrm{i}}$ PubMed $\mathrm{G}$

8. Roger VL, Go AS, Lloyd-Jones DM, Adams RJ, Berry JD, Brown TM, et al. Heart disease and stroke statistics 2011 update: a report from the American Heart Association. Circulation 2011;123(4):e18-209. CrossRef 圈 PubMed 因

9. Holmes MAP, Puckett EM. The Justus-Warren Heart Disease and Stroke Prevention Task Force and the North Carolina plan to prevent heart disease and stroke. N C Med J 2012;73(6):457-60. PubMed 园

10. Acker JE 3d, Pancioli AM, Crocco TJ, Eckstein MK, Jauch EC, Larrabee H, et al. Implementation strategies for emergency medical services within stroke systems of care: a policy statement from the American Heart Association/American Stroke Association Expert Panel on Emergency Medical Services Systems and the Stroke Council. Stroke 2007;38(11):3097-115. CrossRef 恩 PubMed 园

11. Alberts MJ, Latchaw RE, Jagoda A, Wechsler LR, Crocco T, George MG, et al. Revised and updated recommendations for the establishment of primary stroke centers: a summary statement from the Brain Attack Coalition. Stroke 2011;42(9):2651-65. CrossRef 圈 PubMed 圈

12. Williams I, Mears G, Raisor C, Wilson J. An emergency medical services toolkit for improving systems of care for stroke in North Carolina. Prev Chronic Dis 2009;6(2):A67. PubMed 圈

13. Mears GD, Pratt D, Glickman SW, Brice JH, Glickman LT, Cabañas JG, et al. The North Carolina EMS Data System: a comprehensive integrated emergency medical services quality improvement program. Prehosp Emerg Care 2010;14(1):85-94. CrossRef 国 PubMed 圈

14. LaCombe DM, Gordon DL, Issenberg SB, Vega A, Brocato C, Siegel M, et al. Stroke on the mend. JEMS 2000;25 (10):32-41. PubMed 圈

15. Prehospital Medical Information (PreMIS). Chapel Hill (NC): EMS Performance Improvement Center, North Carolina Office of EMS. https://www.emspic.org/applications/premis. Accessed October 12, 2012.

16. Credentialing Information System (CIS). Chapel Hill (NC): EMS Performance Improvement Center, North Carolina Office of EMS. https://www.emspic.org/applications/cis. Accessed March 15, 2012.

17. US Office of Management and Budget. Update of statistical area definitions and guidance on their uses. Washington (DC): Executive Office of the President; 2009. http://www.whitehouse.gov/sites/default/files/omb/assets/bulletins/b1o-02.pdf. Accessed August 4, 2012.

18. Abdullah AR, Smith EE, Biddinger PD, Kalenderian D, Schwamm LH. Advance hospital notification by EMS in acute stroke is associated with shorter door-to-computed tomography time and increased likelihood of administration of tissue-plasminogen activator. Prehosp Emerg Care 2008;12(4):426-31. CrossRef 圈 PubMed 圈 
19. Patel MD, Rose KM, O’Brien EC, Rosamond WD. Prehospital notification by emergency medical services reduces delays in stroke evaluation: findings from the North Carolina Stroke Care Collaborative. Stroke 2011;42(8):22638. CrossRef圈 PubMed 圈

20. McKinney JS, Mylavarapu K, Lane J, Roberts V, Ohman-Strickland P, Merlin MA. Hospital prenotification of stroke patients by emergency medical services improves stroke time targets. J Stroke Cerebrovasc Dis 2013;22 (2):113-8. CrossRef 图 PubMed 圈

21. Shaeffer Z, Gohdes D, Legler J, Taillac P, Larsen B. Monitoring prehospital stroke care in Utah to assess the feasibility of using EMS data for surveillance. Prev Chronic Dis 2009;6(4):A137. PubMed 因

22. McNamara MJ, Oser C, Gohdes D, Fogle CC, Dietrich DW, Burnett A, et al. Stroke knowledge among urban and frontier first responders and emergency medical technicians in Montana. J Rural Health 2008;24(2):189-93. CrossRef 国 PubMed 圈

23. Oser CS, McNamara MJ, Fogle CC, Gohdes D, Helgerson SD, Harwell TS. Educational outreach to improve emergency medical services systems of care for stroke in Montana. Prehosp Emerg Care 2010;14(2):259-64. CrossRef 国 PubMed 圈

24. Shultis W, Graff R, Chamie C, Hart C, Louangketh P, McNamara M, et al. Striking rural-urban disparities observed in acute stroke care capacity and services in the Pacific Northwest: implications and recommendations. Stroke 2010;41(10):2278-82. CrossRef 图 PubMed 圈

25. Williams I, Valderrama AL, Bolton P, Greek A, Greer S, Patterson DG, et al. Factors associated with emergency medical services scope of practice for acute cardiovascular events. Prehosp Emerg Care 2012;16(2):189-97.

CrossRef 圈 PubMed 圈

\section{Tables}

Table 1. Characteristics of Emergency Medical Services (EMS) Stroke-Care Capacity Among 98 EMS Systems, North Carolina, 2012

\begin{tabular}{|l|r|}
\hline \multicolumn{1}{|c|}{ Education and Training } & \multicolumn{1}{|}{ Survey Resultsa } \\
\hline Stroke training provided in past 2 years (95) \\
\hline Median (IQR) hours of stroke training provided in past 2 yearsb & $7.0(4.0-10.0)$ \\
\hline Frequency of stroke trainingb & $21(23)$ \\
\hline More than once per year & $47(51)$ \\
\hline Once per year & $21(23)$ \\
\hline Every 2 or more years & $3(3)$ \\
\hline Only when initially certified & $74(80)$ \\
\hline Stroke educational topics covered in trainingb, c & $92(100)$ \\
\hline Risk factors & $72(78)$ \\
\hline Signs and symptoms & $87(95)$ \\
\hline Pathophysiology & $61(66)$ \\
\hline Scale or screening tool & $50(54)$ \\
\hline Thrombolytic therapy & \\
\hline All 5 stroke educational topics coveredb & \\
\hline Format of stroke training sessionsb, c & $91(99)$ \\
\hline Classroom & $41(45)$ \\
\hline Online & $21(23)$ \\
\hline DVD or video & \\
\hline &
\end{tabular}




\begin{tabular}{|c|c|}
\hline Domains and Measures & Survey Resultsa \\
\hline \multicolumn{2}{|c|}{ Lights and sirens are used while transporting suspected stroke patients } \\
\hline Yes & $30(31)$ \\
\hline No & $10(10)$ \\
\hline Choice made by crew & $58(59)$ \\
\hline Validated stroke scale or screening tool is usedd & $94(96)$ \\
\hline \multicolumn{2}{|l|}{ Stroke scale or screening tool identified as being usedc, e } \\
\hline Los Angeles Prehospital Stroke Screen & $62(66)$ \\
\hline Cincinnati Prehospital Stroke Scale & $49(52)$ \\
\hline Miami Emergency Neurologic Deficit examination & $17(18)$ \\
\hline \multicolumn{2}{|c|}{ Frequency of communicating stroke scale or screen results to destination hospitale } \\
\hline Always & $43(46)$ \\
\hline Very often & $44(47)$ \\
\hline Sometimes & $5(5)$ \\
\hline Rarely & $2(2)$ \\
\hline Never & 0 \\
\hline \multicolumn{2}{|c|}{ Frequency of using a written plan to determine destination hospitalf } \\
\hline Always & $47(49)$ \\
\hline Very often & $37(39)$ \\
\hline Sometimes & $6(6)$ \\
\hline Rarely & $5(5)$ \\
\hline Never & $1(1)$ \\
\hline Policy exists to notify hospital in advance if stroke suspected & $96(98)$ \\
\hline
\end{tabular}

a All values are numbers (percentages) unless otherwise indicated.

b Of the 93 EMS systems that provided stroke training in past 2 years; 1 system did not answer this question.

c Categories are not exclusive.

d Validated stroke scales and screens identified in the survey were the Los Angeles Prehospital Stroke Screen (3), the

Cincinnati Prehospital Stroke Scale (2), and the Miami Emergency Neurologic Deficit examination (14).

e Among the 94 EMS systems that used a validated stroke scale or screen.

f Two systems did not answer this question.

Table 2. Changes in Emergency Medical Services (EMS) Stroke-Care Capacity by 70 EMS Systems From 2001 to 2012, North Carolinaa

\begin{tabular}{|c|c|c|c|c|c|}
\hline Domains and Measures & $\begin{array}{c}2001 \\
\text { Survey, \% }\end{array}$ & $\begin{array}{c}2012 \\
\text { Survey, \% }\end{array}$ & $\begin{array}{l}\text { Absolute Change, } \\
\text { Percentage Points }\end{array}$ & $\begin{array}{c}\text { Relative } \\
\text { Change, \% }\end{array}$ & $\begin{array}{c}P \\
\text { Valueb }\end{array}$ \\
\hline \multicolumn{6}{|c|}{ Education and Training } \\
\hline $\begin{array}{l}\text { Stroke training provided in past } 2 \\
\text { years }\end{array}$ & 90 & 97 & 7 & 8 & .18 \\
\hline $\begin{array}{l}\text { Median no. of hours of stroke } \\
\text { training provided in past } 2 \text { yearsc }\end{array}$ & 4.0 & 6.0 & 2.0 & 50 & $.08 \mathrm{~d}$ \\
\hline \multicolumn{6}{|l|}{ Stroke topics covered in trainingc } \\
\hline Risk factors & 81 & 77 & -4 & -5 & .70 \\
\hline Signs and symptoms & 89 & 97 & 9 & 10 & .11 \\
\hline
\end{tabular}


Preventing Chronic Disease | Emergency Medical Services Capacity for Prehospital Str... Page 10 of 10

\begin{tabular}{|l|r|r|r|r|r|}
\hline Domains and Measures & $\begin{array}{c}\mathbf{2 0 0 1} \\
\text { Survey, \% }\end{array}$ & $\begin{array}{c}\mathbf{2 0 1 2} \\
\text { Survey, \% }\end{array}$ & $\begin{array}{c}\text { Absolute Change, } \\
\text { Percentage Points }\end{array}$ & $\begin{array}{c}\text { Relative } \\
\text { Change, \% }\end{array}$ & $\begin{array}{c}\boldsymbol{P} \\
\text { Valueb }\end{array}$ \\
\hline Pathophysiology & 81 & 74 & -7 & -9 & .36 \\
Scale or screening tool & 61 & 93 & 31 & 51 & $<.001$ \\
Thrombolytic therapy & 55 & 65 & 10 & 18 & .25 \\
4 Basic stroke educational topics & 54 & 67 & 13 & 24 & .12
\end{tabular}

coveredc, e

\section{Practices and Policies}

Suspected stroke patients transported with lights and sirens on, \%

\begin{tabular}{|c|c|c|c|c|c|}
\hline Yes & 11 & 31 & & \multirow{3}{*}{ NA } & \multirow[b]{2}{*}{$.85^{f}$} \\
\hline No & 17 & 9 & & & \\
\hline Choice made by crew & 71 & 60 & & & \\
\hline \multicolumn{6}{|c|}{ Validated stroke scale or screening tool usedg } \\
\hline Yes & 23 & 96 & 72 & 312 & $<.001$ \\
\hline \multicolumn{6}{|c|}{ Policy to notify hospital in advance if stroke suspected } \\
\hline Yes & 71 & 100 & 29 & 40 & $-\mathrm{h}$ \\
\hline
\end{tabular}

Abbreviations: NA, not applicable.

a Although 98 EMS systems responded to the 2012 survey, we had 2001 survey data (5) for only 70 systems. Units indicated in column headings apply to all data in column, except for data on number of hours of stroke training.

b Determined by 2-sided McNemar exact test unless otherwise indicated.

c Systems not providing stroke training were recorded as 0 hours of training provided and no educational topics covered.

d Determined by Wilcoxon rank sum test.

e Basic topics were stroke risk factors, signs and symptoms, pathophysiology, and scale or screening tool. Not included was thrombolytic therapy.

f Determined by Fisher exact test.

g Validated stroke scales and screens named on the survey were the Los Angeles Prehospital Stroke Screen (3), the Cincinnati Prehospital Stroke Scale (2), and the Miami Emergency Neurologic Deficit examination (14).

h Statistic not computed because 2012 data had only 1 response level.

The opinions expressed by authors contributing to this journal do not necessarily reflect the opinions of the U.S. Department of Health and Human Services, the Public Health Service, the Centers for Disease Control and Prevention, or the authors' affiliated institutions.

The RIS file format is a text file containing bibliographic citations. These files are best suited for import into bibliographic management applications such as EndNote 圈, Reference Manager 国, andProCite 圈. A free trial download is available at each application's web site.

For Questions About This Article Contact pcdeditor@cdc.gov

Page last reviewed: September 10, 2013

Page last updated: September 10, 2013

Content source: National Center for Chronic Disease Prevention and Health Promotion

Centers for Disease Control and Prevention 1600 Clifton Rd. Atlanta, GA 30333, USA

800-CDC-INFO (800-232-4636) TTY: (888) 232-6348 - Contact CDC-INFO

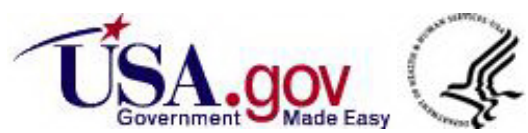

